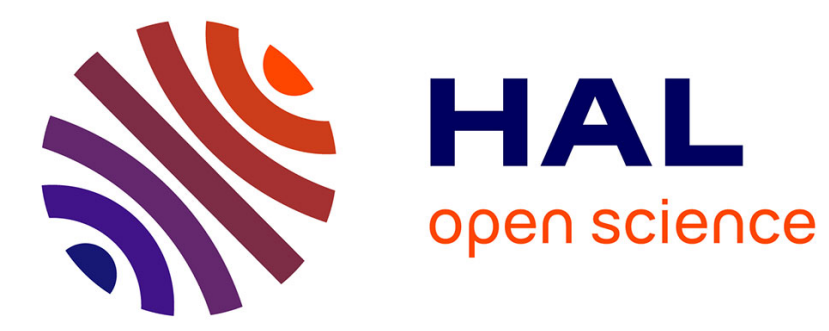

\title{
Le débat français : une toile d'arguments moraux pour un acte controversé \\ Marie Gaille
}

\section{To cite this version:}

Marie Gaille. Le débat français : une toile d'arguments moraux pour un acte controversé. Les Cahiers de la justice, 2016, 2, pp. 289-302. halshs-01453054

\section{HAL Id: halshs-01453054 \\ https://shs.hal.science/halshs-01453054}

Submitted on 2 Feb 2017

HAL is a multi-disciplinary open access archive for the deposit and dissemination of scientific research documents, whether they are published or not. The documents may come from teaching and research institutions in France or abroad, or from public or private research centers.
L'archive ouverte pluridisciplinaire HAL, est destinée au dépôt et à la diffusion de documents scientifiques de niveau recherche, publiés ou non, émanant des établissements d'enseignement et de recherche français ou étrangers, des laboratoires publics ou privés. 


\section{Le débat français : une toile d'arguments moraux pour un acte controversé}

Marie Gaille

\section{Article paru dans les Cahiers de la justice, 2016/12, p. 289-302}

\section{Version avant épreuve}

Le débat français sur la gestation pour autrui : une toile d'arguments moraux pour un acte controversé

Marie GAILLE

Marie Gaillé, directrice de recherche en philosophie au CNRS, rattachée à SPHERE (UMR 7219, CNRSUniversité Paris Diderot, USPC)

Résumé : Cette contribution propose une analyse critiquede la discussion morale développée en France autour de la GPA durant les quarante dernières années.Elle s'appuiesur les avis éthiques émis par divers comités, ainsi quesur lesessais philosophiques ou politiques qui s'y rapportent. Par-delà l'évocation des arguments mobilisés, dont certains renvoient à des principes énoncés comme indiscutables et prévalents (non instrumentalisation de la personne, solidarité procréative, liberté individuelle), elle suggère de revisiter les enjeuxqui sont sousjacents pour mieux en apprécier la portée et les limites. 
En France, depuis bientôt une quarantaine d'années ${ }^{1}$, la gestation pour autrui estun sujet qui suscite l'expression de désaccords apparemment insurmontables dansdifférents espaces de discours ${ }^{2}$. Comme pour certaines questions dites de «bioéthique»,cette dissonance a pour caractéristique d'être énoncée à travers des arguments quitraversent les frontières entre les disciplines (notamment médecine, droit, philosophie,sociologie, anthropologie, psychologie), mais aussi entre l'espace académique etscientifique et le débat public, politique, législatif.

La discussion menée sur la gestation pour autrui y a aussi pour particularité de seconcentrer sur une pratique qui implique des individus - un couple désireux d'avoir unenfant et une femme qui accepte de porter un enfant qui deviendra leur enfant - et nondes individus et un Etat soucieux du niveau démographique de sa population, voire de laqualité de ses gènes. Lorsque André Gorz imagine, en 1988, l'institution d'un «salairematernel » par un Etat désireux de «se faire fournir des enfants», il souligne ladimension utopique de cette perspective - utopie négative dont l'histoire donnecependant quelques exemples de réalisation: «On retrouve là Le meilleur des mondes d'Aldous Huxley, mais aussi les pratiquesdu Ille Reich: la maternité est interdite (par stérilisation) aux femmes dont laprogéniture ne serait pas conforme aux normes d'eugénisme; inversement, laprocréation est encouragée dans les 'fontaines de vie' (Lebensborne) où de jeunesfemmes de type nordique se font engrosser par de jeunes SS afin de fournir au Reich etau Führer les futures élites raciales. Les enfants nés dans ces centres de procréationn'ont jamais connu leurs parents $»^{3}$.

Une troisième caractéristique saillante de cette discussion est de se concentrersur des questionnements juridiques et moraux. Deux questions principales structurentcette discussion : peut-on justifier le recours à la gestation pour autrui d'un point de vuemoral ? Peut-on la légaliser, et le cas échéant quel cadre juridique lui donner ? Untroisième élément de discussion complète ce second volet. Il a trait au statut juridiqueconféré, en France, aux enfants nés grâce au recours à la gestation pour autrui.

Ce qui est proposé ici est l'examen des arguments mobilisés pour répondreà ces interrogations, puis une analyse critique de ces derniers ; l'idée étant de se concentrer surles arguments avancés au plan moral, c'est-à-dire perçus par ceux qui lesénoncent et en débattent comme relevant de la réflexion sur le bien, le bon, le juste. Comme le souligne l'anthropologue R. Massé, toutes lesjustifications des actions humaines ne sont pas et n'ont pas nécessairement à être morales en ce sens ${ }^{4}$. Or, il est remarquable que les «balises », selon son expression, permettant de juger de lapratique de la gestation pour autrui, sonttrès souvent associées « au bien, au bon, au juste ».

\footnotetext{
${ }^{1}$ Le débat a émergé notamment avec la publicité faite, au début des années 1980, à l'organisation par deux associations de rencontres entre des couples hétérosexuels infertiles et de femmes susceptibles d'être génitrices et gestatrices pour ces couples.

${ }^{2}$ C'est par commodité et en rapport avec l'usage le plus fréquent que je parle ici de gestation pour autrui. Il serait sans doute plus approprié de parler de «procréation pour autrui », expression plus large de signification, susceptible de recouvrir tous les cas de figure : celui où la mère porteuse désigne la femme qui donne un ovocyte et porte l'enfant; celui où elle est gestatrice, l'ovocyte provenant ou de la mère d'intention ou d'une autre femme.

${ }^{3}$ A. Gorz, Métamorphoses du travail- Critique de la raison économique, Paris, Gallimard Folio essais, 1988,2, Ill, 4bis, p. 243.

${ }^{4}$ R. Massé, Anthropologie de la morale et de l'éthique, Les Presses de l'Université de Laval, 2015, p. 1.
} 
Pour rendre compte et analyser ces arguments, on s'est appuyé sur des textes quiont pour vocation de contribuer à la discussion publique sur un plan éthique (avis issus de comités, de sociétés savantes, essais philosophiques et politiques). Danscette contribution, seront laissés de côté les arguments de nature juridique (notamment,l'indisponibilité de l'état des personnes, l'interdiction de la commercialisation du corpshumain, la définition de la maternité par accouchement) et la question de la capacité dudroit français à se réformer en vue de légaliser la gestation pour autrui ${ }^{5}$. Ces deuxaspects ne sont pas moins importants pour l'analyse du débat sur la gestation pourautrui, mais leur commentaire excède le domaine de compétences qui est le nôtre.

\section{Arguments moraux pro et contra la gestation pour autrui}

Des « impératifs catégoriques » antagonistes

La discussion fait place de façon importante à une réflexion en terme de principesmoraux destinés à réguler les relations interindividuelles. Ceux-ci s'énoncent sur lemode de $l^{\prime}$ « impératif catégorique ${ }^{6}$, au sens donné par Kant à ce terme, c'est-à-dired'une loi (morale) pratique inconditionnelle, qui surpasse tout autre principe, norme ouvaleur possible, toute prise en compte d'un désir, quelle que soit l'importance accordée àcelui-ci, ou d'une situation de fait. Un premier principe de ce genre est le refus del'instrumentalisation de la personne humaine et de son corps. Cette instrumentalisationest dénoncée avec d'autant plus de vigueur qu'elle est aggravée tout d'abord des dangersinhérents à toute grossesse pour la femme qui porte l'enfant à naître ${ }^{7}$. Elle l'est en outrepar les risques psychiques supposés, sur le court et le long terme, pour la mère porteuse,sa famille et l'enfant à naître. Les parents d'intention feraient fi trop facilement des lienstissés entre eux in utero afin de satisfaire leur propre désir d'enfant.

L'association de la gestation pour autrui à une forme d'instrumentalisationinacceptable est particulièrement forte lorsqu'elle est sous-tendue par une conceptionde la maternité comme un tout, de la conception à l'éducation en passant par la gestationet l'accouchement.

La discussion éthique, en France, fait place à une seconde position de principequi, à l'inverse, conduit à se prononcer en faveur de la gestation pour autrui : au nom dela solidarité à l'égard de femmes affectées par une infertilité d'origine utérine(notamment suite à un cancer) ou qui n'ont plus d'ovaires, la gestation pour autrui,médicalement organisée, est - au nom de cette solidarité - la réponse qui s'impose pourrépondre au désir d'enfant de ces femmes et de leurs conjoints ${ }^{8}$. Bien que le Rapport Terra Nova Accès à la parenté -Assistance

\footnotetext{
${ }^{5}$ Pour une analyse critique de cet aspect, voir M. Iacub, L'empire du ventre. Pour une autre histoire de la maternité, Paris, Fayard, 2004.

${ }^{6}$ E. Kant, Fondements de la métaphysique des mours, trad. de V. Delbos, Paris, Vrin, 1957, p. 134 et p. 99.

${ }^{7}$ Avis 110, Comité Consultatif national d'éthique, Problèmes éthiques soulevés par la gestation pour autrui (2010) : «Or les risques médicaux, y compris vitaux, encourus par la femme enceinte et l'enfant lors de la GPA sont réels et ont été rappelés de manière exhaustive par l'Académie de médecine ; ils englobent en particulier les cas de grossesses multiples et de prématurité qui seraient encourus dès lors qu'on ne se limiterait pas à transférer un seul embryon, mais aussi la réalisation d'une césarienne ou d'une intervention pour une hémorragie de la délivrance. (...) Même si grossesse et délivrance se passent normalement, les grossesses et les accouchements répétés éprouvent le corps des femmes et peuvent avoir des répercussions sur leur santé ultérieure. »>, p. 7.

${ }^{8}$ L'argument est rappelé dans l'Avis 110 du Comité consultatif national d'éthique.
} 
médicale à la procréation et adoption n'utilise pas ce terme, il formule une proposition de loiau sujet de la gestation pour autrui proche, dans son esprit, de cette position solidaire. Il prône en effet la légalisation de la gestation pour autrui «afin que les femmes chez qui se trouventdiagnostiquée une impossibilité pathologique d'assurer la gestation puissent enbénéficier $\gg)^{9}$.En 2010 , le Comité consultatif national d'éthique envisage également la légalisation de la gestation pour autrui, dans certaines limites, comme une réponse de la société à une « injustice » :

«La GPA apparaît comme une solution à un problème physique et psychiquedouloureux. L'infertilité d'origine utérine est souvent perçue comme d'une particulière injustice. Elle touche des femmes qui «ont tout pour être mère» sauf l'utérus, tandis que celles qui ont n'ont plus d'ovaires, mais ont encore un utérus, peuvent bénéficier du don d'ovocyte, et que les femmes dont les conjoints sont infertiles peuvent bénéficier du don de sperme ${ }^{10}$.

C'est encore l'argument de la solidarité qu'invoque, en 2014, l'Académie de médecine,lorsqu'elle évoque la souffrance ressentie par les couples infertiles, leur vécu d'unesituation d'injustice dans un contexte où l'adoption demeure compliquée, et pas toujoursdésirée, et où il n'existe pas d'alternative thérapeutique ${ }^{11}$.

Une troisième position de principe s'exprime, bien différente des deux précédentes, en faveur de la gestation pour autrui. Dans le sillage de J. S. Mill, le philosophe R.Ogien a présenté une argumentation qui combine un critère demoralité (l'absence de nuisance à autrui) et un impératif catégorique politique (la libertéindividuelle politique) : la liberté des individus à mener la vie de leur choix, dès lorsqu'ils ne nuisent pas à autrui, doit absolument primer sur toute autre norme pourréguler les relations entre les citoyens et l'Etat.

Selon lui, le libéralisme (moral etpolitique) dont se réclame la société française est très insatisfaisant, voire hypocrite àses yeux et les libertés individuelles y sont menacées par la montée contemporaine de la «pensée conservatrice $»^{12}$. Compte tenu de ce contexte, la mission de la philosophie estavant tout de «démoraliser »les questions et énoncer les « raisons philosophiques derésister à l'influence de cette pensée, et de lui préférer des idéaux politiques etlibertaires ${ }^{13}$. R. Ogien rejette en ce sens l'idée selon laquelle le droit devrait être fondésur des valeurs morales. En réalité, il n'entend pas dépouiller le droit de toute vocationnormative, dans la mesure où il entend faire de la liberté individuelle négative l'objetprincipal de l'action politique, juridique et institutionnelle. Sa thèse est en effet qu'un État doit se doter d'une législation fondée sur le principe de la liberté négative entenduecomme «conception minimaliste de la liberté politique »:

«Pratiquement, elle 'cette conception' trace autour de chaque individu un largepérimètre de protection qui doit le mettre à l'abri non seulement de la servitude et de l'oppression, mais aussi de toutes les formes de persécution, et de toutes les tentatives d'extermination. À l'intérieur de ce périmètre, elle laisse chacun libre de faire ce qu'il veut de sa propre vie ${ }^{14}$.

\footnotetext{
${ }^{9}$ Rapport Accès à la parenté - Assistance médicale à la procréation et adoption, coordonné par G. Delaisi de Parseval et V. Depadt-Sebag pour Terra Nova (2010), p. 78.

${ }^{10}$ Avis 110 du Comité consultatifnational d'éthique, p. 18.

11 «La Gestation pour autrui au regard du mariage entre personnes de même sexe », Bull. Acad. Natle Méd., 2014, 198, nº-5, p. 917-950, séance du 27 mai 2014, http://wWw.academie-medecine.fr/articles-dubulletin/publication/?idpublication=100319 (consulté le 13 février 2016).

${ }^{12}$ R. Ogien, L'Etat nous rend-t-il meilleurs? Essai sur la liberté politique, Paris, Gallimard, 2013, p. 11.

${ }^{13}$ Ibid., p. 12.

${ }^{14}$ Ibid., p. 25.
} 
Les implications de ce positionnement sur la famille tel que le droit devrait laconcevoir sont de son point de vue sans ambiguïté :la procréation, comme la fin de vie, affirme-t-il, sont des «domaines si intimes » qu'il convient de ne pas intervenir pour réguler les décisions d'une quelconque manière, dès lors que l'action engagée ne nuitpas à autrui ${ }^{15}$. Entre autres choses, il n'existe aucune raison valable d'interdire lemariage entre personnes de même sexe ${ }^{16}$, ni de refuser aux couples de même sexel'accès à la procréation médicalement assistée et à la gestation pour autrui dès lorsqu'une femme consent à devenir la mère porteuse d'un enfant pour des parentsd'intention ${ }^{17}$.

\section{La pauvreté des mères porteuses}

La discussion éthique ne repose pas seulement, en France, sur une réflexion deprincipe, moral ou politique. Elle n'est pas toujours « hors sol », coupée de toutedimension sociologique, dans la mesure où elle fait également une place importante àl'analyse des caractéristiques socio-économiques de la gestation pour autrui ${ }^{18}$. Celle-ciconduit à donner une place importante à la question de l'exploitation de situationsd'inégalités économiques entre mère porteuse et parents d'intention, et débouche, le plussouvent, sur un positionnement éthique critique à l'égard de la gestation pour autrui.Dans ce cas, il ne s'agit pas, ou pas seulement, de dénoncer l'instrumentalisation ducorps d'autrui, mais d'accuser les parents d'intention de profiter d'un avantageéconomique comparatifpour réaliser leur désir d'enfant.

De fait, on a pu constater que des femmes, dans différents pays du monde,consentaient à être, en échange d'une certaine rétribution, des mères porteuses, mêmesi certaines enquêtes documentent des décisions de mères porteuses qui n'apparaissentpas être dans le besoin économique. Dans les enquêtes dont nous disposons pourconnaître leur expérience, elles soulignent qu'une vie de travail n'aurait jamais permisde gagner la même somme que celle liée à une gestation pour autrui ${ }^{19}$. De là est tirée l'idée qu'elles n'auraient jamais consenti à porter un enfant pour autrui, voire à donnerleurs ovocytes, si leurs conditions socio-économiques avaient été meilleures. Lagestation pour autrui est alors perçue comme un acte moralement inacceptable, parce qu'ilconsiste à profiter d'un rapport d'inégalités économiques.

\section{Analyse critique}

\footnotetext{
${ }^{15}$ Ibid., p. 238.

${ }^{16}$ Ibid., p. 60-62.

${ }^{17}$ D'où une critique virulente du «modèle familial » qui sous-tend selon lui la loi de Bioéthique de 2011, «censé être le seul concevable, sans autre raison que des préjugés culturels et des hypothèses psychologiques plutôt fumeuses sur l'intérêt de l'enfant », Ibid., p. 228-229.

${ }^{18}$ De ce point de vue, on peut nuancer le jugement de D. Mehl à l'égard de cette discussion bioéthique française, qui porte selon elle avant tout sur des principes : «Pourquoi, avant de réprimer, ne pas écouter, tant les parents intentionnels que les mères porteuses elles-mêmes ? Pourquoi légiférer au nom de principes jamais confrontés au réel plutôt que d'aborder pragmatiquement un domaine où règnent les incertitudes mais dont les acteurs ont pensé leur projet et son en mesure de transmettre leur expérience ? Et qui sont, aujourd'hui, victimes, eux et leurs enfants, d'une loi votée sans qu'ils aient été conviés à son élaboration ", Enfants du don - Procréation médicalement assistée - parents et enfants témoignent, Paris, Robert Laffont, 2008, p. 238.

${ }^{19}$ Sh. Rudrappa, «Des ateliers de confection aux lignes d'assemblage des bébés. Stratégies d'emploi parmi des mères porteuses à Bangalore, Inde », (trad. M. Forest), Cahiers du Genre, 2014/1, n $^{\circ}$ 56, p. 59-86. DOI 10.3917/cdge.056.0059.
} 
Les différents arguments évoqués ci-dessus n'occupent pas tous une placeéquivalente dans la discussion française sur la moralité/immoralité de la gestation pourautrui et sur le bien-fondé de sa légalisation.

Le premier argument de principe lié à laquestion de l'instrumentalisation est prédominant. Avancé pour dénoncer l'immoralitéde la gestation pour autrui, il s'articule et se combine avec divers éléments inscrits dansle droit français: le principe de noncommercialisation du corps humain, la définition dela maternité par accouchement et le principe de l'indisponibilité des personnes.L'organisation juridique et les options morales exprimées en dehors du droit se renforcentmutuellement en faveur d'un rejet de la gestation pour autrui. Dans un contexte social etune organisation du système de santé marqués par l'idée de la solidarité, comme c'est lecas en France, l'argument d'une nécessaire réponse à une situation d'injustice et desouffrance rencontre un écho important. Il est cependant moins puissant à ce jour que lepremier argument. Enfin, le troisième argument de principe, lié à l'idéal de libertépolitique et à une conception «minimaliste» de la morale, apparaît minoritaire, à la foispar rapport à la force du premier argument et à la prise en compte de l'exploitation desituations de pauvreté.

Même s'il est «particulièrement naïf de prétendre échapper au dilemme » ${ }^{20}$ et deformuler des arguments qui emporteraient une pleine et entière conviction sur lagestation pour autrui, on voudrait ici proposer une analyse critique de ces éléments dediscussion, et dégager leur portée et limite.

Revenons tout d'abord sur l'impératifcatégorique de ne pas instrumentaliser la personne et son corps, en l'occurrence, celuide la mère porteuse et peut-être aussi de l'enfant à naître. La question qui se pose toutd'abord à son sujet est celle de la nature de cette instrumentalisation. En effet, lagestation pour autrui est envisagée dans cette discussion comme un acte consentie. Lagestation pour autrui ne s'apparente pas, de ce point de vue, à une situation d'esclavage.Si l'on reprend à la lettre la formulation de l'impératif pratique proposée par Kant, laqualification de la gestation pour autrui comme instrumentalisation de la personne perd son évidence : «l'impératif pratique sera donc celui-ci : agis de telle sorte que tu traitesl'humanité aussi bien dans ta personne que dans la personne de tout autre toujours enmême temps comme unefin, etjamais simplement comme un moyen $»^{21}$.

Jamais simplement comme un moyen. Peut-on dire qu'une personne qui consentest traitée par autrui simplement comme un moyen ? Cela paraît peu recevable. Il estpeu convaincant de manier cet impératif catégorique pour dénoncer l'immoralité desparents d'intention. En revanche, ce qui devient problématique dans cette perspective est peut-êtrela décision de la mère porteuse. En consentant à porter un enfant qu'elle n'élèvera pas,ne fait-elle pas d'elle-même un simple moyen ? Ne remet-elle pas en cause « la fin » qu'elle est pour ellemême et pour autrui, et à travers elle, l'humanité comme fin ?Laquestion se pose.

Mais la formuler, n'est-ce pas faire fi de liberté de décisiond'une personne ? N'est-ce pas se montrer abusivement paternaliste à leur égard? Certes,comme le souligne le philosophe du droit américain R. Dworkin, J. S. Mill a lui-mêmeindiqué une limite à sa critique du paternalisme: personne ne devrait être autorisé àconsentir à son propre esclavage,

\footnotetext{
${ }^{20}$ I. Théry et A.-M. Leroyer, Filiation, origines, parentalité - le droit face aux nouvelles valeurs de responsabilité générationnelle, Paris, O. Jacob, 2014, p. 215.

${ }^{21}$ Ibid., p. 150
} 
car une telle décision remet en cause son autonomieprésente et future $?^{22}$ Cependant, on ne peut dire que la décision de consentir à porter unenfant pour autrui remet en cause l'autonomie présente et future de la mère porteuse.A-t-on dès lors de bonnes raisons d'empêcher une femme de devenir mèreporteuse ?

Peut-être se trouvent-elles du côté des effets de la gestation pour autrui sur lamère porteuse, sa famille et l'enfant à naître. Le cas de «Baby $\mathrm{M}$ », discutée par laphilosophe américaine B. Steinbock, illustre la situation tragique d'une mère porteusequi, après la naissance de l'enfant, n'a pas voulu s'en séparer ${ }^{23}$. Cependant, contre une visionpessimiste des dégâts psychiques causés par une gestation pour autrui, plusieursenquêtes contemporaines ont mis l'accent sur le sentiment d'accomplissement desmères porteuses, la valeur morale qu'elles accordent à leur acte (le don de la vie,l'altruisme, notamment à l'égard de couples infertiles) ${ }^{24}$. Le même souci des effets à longterme des liens tissés in utero s'exprime au sujet de l'enfant à naître ${ }^{25}$. Le rapport TerraNova propose de cet enjeu une analyse nuancée qui se veut rassurante :

«On sait par ailleurs que les émotions de la fin de la grossesse, qu'il s'agissed'euphorie ou de dépression, secrètent des molécules qui franchissent le filtreplacentaire: le bébé hérite non seulement des gènes de la mère génétique, mais aussid'une partie de l'histoire de la mère gestatrice. Il n'est donc pas question de nier le rôle dechacune, particulièrement de la gestatrice, au regard de l'équilibre de l'enfant, maisd'éviter toute mythification des rapports entretenus par la gestatrice et l'enfant qu'elle a porté durant neuf mois. (...) Sans ignorer ou minimiser ces réalités, nous savonsaujourd'hui, tant d'après les données de la psychopathologie périnatale que d'aprèscelles de l'adoption, qu'un enfant porté par une femme qui n'est pas la mère d'intentionsera capable, par déplacement, de faire un transfert sur d'autres adultes, à condition queceux-ci s'y prêtent de façon adéquate ${ }^{26}$.

On sait peu de choses sur les effets de la gestation pour autrui sur les membres de la famille de lamèreporteuse ${ }^{27}$.

Finalement, surcette question, beaucoup d'affirmationssont encore en partiede l'ordrede la spéculation. L'élaboration d'un point de vue fondé à son propos requiert des enquêtes plus poussées que celles dont nous disposonsactuellement, en France comme à l'étranger, d'autant qu'on peut s'interroger, comme yinvite la sociologue A. Pande, sur la

\footnotetext{
${ }^{22}$ G. Dworkin, "Paternalism", in R. A. Wasserstrom (éd.), Morality and the Law, Belmont, California, Wadsworth, 1971.

${ }^{23}$ B. Steinbock, "Surrogate Motherhood as Prenatal Adoption", Law, Medicine and Health Care, 16, 1-2, Spring, 1988, p. 44-50.

${ }^{24}$ Rapport Terra Nova, p. 72.

${ }^{25}$ Voir l'Avis 110 pré-cité du CCNE, qui passe directement d'une interrogation - « on peut néanmoins s'interroger sur ce qui peut persister en lui de cette période de gestation » - à une affirmation sur l'avenir de l'enfant - «Il est donc actuellement difficile d'admettre sans réserve que la GPA préserverait l'avenir de l'enfant ».

${ }^{26}$ Rapport Terra Nova, p. 73-74. J. Merchant évoque des études qui indiquent à ce sujet qu'aux Etats-Unis, « la vaste majorité des parents de ces nouvelles familles ont dit la vérité aux enfants, et ont choisi de garder le contact avec la mère porteuse. Les enfants de ces familles ne diffèrent pas dans leurs rapports avec leurs parents des enfants nés 'traditionnellement', certains même parlant d'un 'plus' dans leurs vies en référence au maintien de contact avec la femme porteuse ». «Une gestation pour autrui «éthique» est possible », Travail, genre et sociétés, 2012/2 n² 28, p. 183-189. DOI: 10.3917/tgs.028.O18, p. 189. Voir S. Golombok, V. Iavda, L. Blake, J.Readings, P. Casey, A. Marks, "Families Created Through Surrogacy :Mother- Child Relationships and Children's Psychological Adjustment at Age 7", Developmental Psychology, 2011, 47/6, p. 1579-1588 ; V. Javda, S. Golombok, L. Blake, J. Readings, P. Casey, "Surrogacy Families Ten Years On : The Relationship with the Surrogate, Decisions Over Disclosure and Children's Understanding of their Surrogacy Origins", Human Reproduction, 2011, 26 (suppl 1): i260-i271.

${ }^{27}$ Cet aspect estdiscuté par B. Steinbock, $<<$ Surrogate Motherhood as Prenatal Adoption », art. cit., p. 49.
} 
portée des témoignages de mères porteuses liés auxnotions de don et d'altruisme. Si l'usage de ces termes permettent à ces femmes deminorer la partie marchande de la gestation pour autrui - tout comme le vocabulaire dela compassion pour les mères d'intention -, ils font selon elle écran aux lesrapports d'inégalités postcoloniaux entre les «sœurs du Sud » (mères porteuses) etcelles du Nord (mères d'intention) ${ }^{28}$ et partant, entretiennent ces rapports.

Revenons maintenant à l'argument lié à l'exploitation de la pauvreté. Si une partiedes femmes qui acceptent d'être mères porteuses le font parce que c'est pour ellel'unique moyen de remédier à leur extrême pauvreté, la gestation pour autrui n'est-ellepas fondée sur un accord moralement problématique ? Sur ce point, l'argument lié àl'exploitation de la pauvreté semble faire d'une pierre deux coups : il remet en cause lasolidité du consentement et fait apparaître les parents d'intention comme des «profiteurs » d'un contexte d'inégalité.

Cet argument appelle plusieurs commentaires. Sur la question du consentement biaisé, sa portéedoit être relativisé. Peut-on, en effet, dévaloriser le choix d'une personne au nomdu fait qu'il est opéré dans un contexte jugé contraint? Cela ne va pas de soi. On peut, aucontraire, défendre l'idée selon laquelle une véritable capacité de choix se maintient,même en contexte fortement contraint. À tout le moins, on peut avancer la thèse quecertaines décisions prises dans des contextes très contraints peuvent être décritescomme partiellement volontaires, ainsi que le suggère Aristote ${ }^{29}$. Dans les faits, quelquesenquêtes étayent cette perspective. Ainsi, S. Rudrappa, à partir d'une enquête de terrainmenée à Bengalore, en Inde, bien que situées dans un contexte d'extrême pauvreté, lesfemmes qui optent pour devenir mères porteuses ne peuvent être décrites de façonunilatérale comme des victimes d'une situation d'inégalités. Elles tirent de ce choix desbénéfices d'ordre financier non négligeables en ce qu'il leur permet d'échapper à «l'enfer » du travail en usine. Elles en retirent aussi la possibilité d'un repositionnementvalorisant pour elles, au sein de leur famille (par rapport à leur mari et à leur belle-famille). Enfin, elles en retirent une satisfaction personnelle, liée au don de la vie et aurôle qu'elles jouent pour les parents d'intention, en leur donnant la possibilité d'éleverun enfant. Les difficultés organisationnelles et psychiques de ce choix ne sont pas niéeset passées sous silence (surveillance des grossesses; accouchement par césarienne; séparation d'avec leur famille pendant neuf mois; lutte intérieure contre les éventuelssentiments d'attachement pour l'enfant porté). Il faut reconnaître que ces femmes font, dans le contexte qui est le leur et ses contraintes propres, comme tout le monde. Ellesévaluent l'avantage d'un acte comparativement à un autre :

« «Çafait mal, mais qu'est-ce que vous voulez répondre à ça ?», déclara Vijaya. Ce àquoi Nagu répliqua: «aucun homme n'est capable de gagner 4000\$ en neufmois et de leremettre entre les mains de safemme. Aucun». À ses yeux, la GPA lui donne davantage depouvoir et d'autonomie précisément parce qu'elle est une femme. Elle lui permet de mettre à contribution son corps pour gagner une somme qu'aucun homme ne

\footnotetext{
${ }^{28}$ A. Pande, "Transnational commercial surrogacy in India: gifts for global sister ?", Reproductive Biomedicine Online, 2011, 23, p. 618-625.

${ }^{29}$ Aristote : «c'est là encore ce qui se produit dans le cas d'une cargaison que l'on jette par-dessus bord au cours d'une tempête: dans l'absolu, personne ne se débarrasse ainsi de son bien volontairement, mais quand il s'agit de son propre salut et de celui de ses compagnons un homme de sens agit toujours ainsi. (...) Volontaires sont donc les actions de ce genre, quoi que dans l'absolu elles soient peut-être involontaires, puisque personne ne choisirait jamais une pareille action en elle-même », Éthique à Nicomaque, trad. J. Tricot, Paris, Vrin, 1987, III, 1, 1109b$1110 \mathrm{a}$, p. 119.
} 
pourraitgagner en seulement neuf mois. C'est donc son sexe qui lui a permis de gagner unesomme importante, une chose jusque-là inimaginable dans son univers social $»^{30}$.

Qu'en est-il de cet argument de l'exploitation si l'on s'intéresse aux parents d'intention? Sur cepoint, la réflexion du philosophe E. Malmqvist fait apparaître que «l'exploitation», ausens où nous l'entendons couramment, n'est que l'un des aspects du problème et peut-être pas le principal point d'achoppement du point de vue moral. Partant de l'idéequ'une personne prend des décisions qui ne peuvent être disqualifiées en raison d'uncontexte contraint, E. Malmqvist estime qu'on ne peut assimiler automatiquementl'exploitation d'une situation d'inégalités à une injustice, a fortiori lorsqu'elle est mutuellement bénéfique et consentie. On pourrait même avancer l'idée selon laquelle lerecours à la gestation pour autrui, améliorant la situation (économique, sociale,familiale) de la mère porteuse vaut mieux que la négligence à l'égard de ses besoins : cette somme dont je dispose, ne devrais-je pas l'investir dans un contrat de gestationpour autrui, afin de réaliser mon désir d'enfant, et par la même, aider une femme pauvre, plutôt que de renoncer à ce désir, et acheter une maison au bord de la mer $?^{31}$ Vue sous cet angle, la gestation pour autrui apparaît être un moindre mal compte tenudes inégalités économiques globales.

Ce point de vue a cependant une portée limitée dès lors que l'on introduit, souligne E. Malmqvist, laquestion de la complicité dans l'injustice ${ }^{32}$. La complicité dans l'injustice, même quandon n'est pas cause de celle-ci, désigne les comportements, choix, attitudes quinourrissent et renforcent des inégalités structurelles moralement inacceptables. Danscette perspective, ceux qui ont recours à une mère porteuse non seulement tirentponctuellement profit des inégalités globales, mais encouragent par leur acte, de façonimplicite, le maintien du statu quo à leur sujet. De la sorte, E. Malmqvist estime que leconsentement mutuel des parents d'intention et d'une mère porteuse, et le bénéfice quecelle-ci en retirent, ne sont pas des raisons suffisamment bonnes pour justifiermoralement la gestation pour autrui :

«Once we recognize that exploitation coexists with complicity when it arisesfrom injustice it becomes less puzzling to think that it can be worse to exploit the globalpoor than to neglect them, even when exploitation is voluntary and makes them better off(...)The point for now is that irrespective of our views on neglect, exploitation can beseriously wrong - even in its mutually beneficial and voluntary form ${ }^{33}$.

Formulé ainsi, l'argument lié à l'exploitation des mères porteuses par les parentsd'intention a une portée morale importante dans la discussion sur la gestation pour autrui. Il paraît convaincant et plus à même de faire pencher la balance en faveur d'undésaveu moral de la gestation pour autrui que l'argument de l'instrumentalisation. Cen'est pas cet argument, pourtant, que l'on retrouve de façon prégnante dans le débatfrançais, même lorsqu'il

\footnotetext{
${ }^{30}$ Sh.Rudrappa, «Des ateliers de confection aux lignes d'assemblage des bébés. Stratégies d'emploi parmi des mères porteuses à Bangalore, Inde », art. cit., p. 72. Dès le début des années 1980, cette critique de l'argument à l'égard du consentement biaisé, a été élaborée cf. J. Robertson, «Surrogatemother : not sonovel after all", Hastings Center Report, 13, 5, 1983, 29.

${ }^{31}$ E. Malmqvist, «Transantional commercial surrogacy, exploitation and the non-worseness claim » (exposé oral présenté dans le séminaire international PLURIGENRE organisé par V. Bourseul et M. Gaille, "Médecine procréative, différence sexuelle et égalité des sexes », Paris, CNRS-Université Paris Diderot, 16 octobre 2015).

${ }^{32} \mathrm{Ch}$. Kutz, Complicity: ethics and law for a collective age, Cambridge University Press, 2000; E. Malmqvist, "Taking advantage ofinjustice", Social Theory and Practice, 2013, 39, 4, 557-580.

${ }^{33}$ E. Malmqvist, «Transantional commercial surrogacy, exploitation and the non-worseness claim », exp. Cit.
} 
s'agit de mettre en cause le primat accordé par certains à laliberté individuelle et au consentement.

Telle qu'elle est ainsi organisée, la discussion française pâtit de plusieursproblèmes auxquels il est souhaitable de remédier afin de la poursuivre dans demeilleures conditions. Elle se déploie, nous l'avons vu, dans une relative ignorance deseffets à long terme de la gestation pour autrui pour la mère porteuse, sa famille etl'enfant à naître, et de la portée que nous devons donner à ces effets dans l'évaluationmorale de la gestation pour autrui ${ }^{34}$.De façon générale, cette situation de relative ignorance est aggravée en France par l'interdiction de la gestation pour autrui, qui rend les enquêtes de terrain/quantitativesdifficiles à concevoir et à mettre en œuvre. Cette ignorance, qui n'est pas propre à laFrance, rend toute affirmation générale douteuse sur la gestation pour autrui ${ }^{35}$. Malheureusement, ce contexte facilite partout l'usage de formules floues, polémiques, parfois insultantes pour les personnes, au sujet de la gestation pour autrui : «mutationanthropologique majeure», «marchandisation du corps humain », « «le corps de la femme» comme« outil de production», «babybusiness », «aliénation biologique », « couveusesindemnisées », «tourisme procréatif » ${ }^{36}$, « dégoût à devoir argumenter $»^{37}$ etc.

Par ailleurs, le cadre de la discussion est très restrictif par rapport à sonextension possible et a des angles morts préoccupants. En principe, le recours à lagestation pour autrui peut être envisagé pour réaliser un désir d'enfant exprimé pourdiverses raisons : par des couples hétérosexuels, dont la femme ne peut porter l'enfant ànaître en raison d'une déficience de la fonction utérine ; par des couples homosexuelsmasculins, qui ont besoin non seulement des ovocytes d'une femme, mais aussi d'unefemme qui accepte d'être leur gestatrice (et qui n'est pas nécessairement celle qui adonné ses ovocytes) ; par des couples hétérosexuels dont la femme, en état de procréeret d'être gestatrice, ne désire pas être enceinte et porter pendant neuf mois l'enfant à naître; par un homme qui désire fonder seul une famille et voudrait recourir à une mèreporteuse, à la fois pour le don d'ovocytes et la gestation, ou d'une femme qui nourrit lemême désir, dispose de sperme, donné ou acheté, et ne peut/veut porter l'enfant.

EnFrance, la discussion porte avant tout sur la première situation. Les réflexions qui fontexception à cet état des choses, et abordent le cas des couples homosexuels, sontd'autant

\footnotetext{
${ }^{34}$ Le travail de D. Mehl, Enfants du don - Procréation médicalement assistée - parents et enfants témoignent (op. cit.) tente de remédier de façon explicite à cette situation d'ignorance, en exposant quelques récits de parents d'intention hétérosexuels (2014). Ce constat d'ignorance peut être étendu au point de vue des citoyenssur la gestation pour autrui, cf. ChPetitfils et M. T. Munoz Sastre, "French Layersones' Views on Surrogate Motherhood - An exploratory study", Psicologica, 2014, 35, 693-702.

${ }^{35}$ O. B. A. van den Akker : «Data fromsurrogatemothers (on psychopathology and motives), fromintended/commissioningmothers (on openness and psychopathology) and fromoffspring (on attachment and openness) are rare. The evaluation of the evidencepresented in thisreview has alsodemonstratedthattherewas a notable lack of theory and experimentstestingappropriatelydefinedmodels. No interventions and few longitudinal studies have been carried out. Virtually all studies, of necessity, usedhighlyselectedsamples, makinggeneralizationsdifficult. », «Psychosocial aspects of surrogatemotherhood», Human Reproduction Update, 13/1, 53-62, 2007.

${ }^{36}$ Voir la critique bienvenue de cette expression par D. Mehl, Enfants du don - Procréation médicalement assistée - parents et enfants témoignent (op. cit.) ou par I. Löwy et al., «Nouvelles techniques reproductives, nouvelle production du genre. Introduction », Cahiers du Genre, 2014/1, n ${ }^{\circ}$ 56, p. 5-18. DOI 10.3917/cdge.056.0005.

${ }^{37}$ S. Agascinski, Le corps en miettes, Paris, Quai Voltaire, 2009.
} 
plus remarquables qu'elles sont rares. Le rapport proposé par G. Delaisi deParseval et V. Depadt-Sebag en 2010 pour Terra nova expose sur des plans spécifiquesleur désaccord à ce sujet et propose les modalités d'une révision de la loi dans cetteperspective «à terme », c'està-dire dans un horizon temporel indéterminé ${ }^{38}$. L'idée d'unrecours à la gestation pour autrui pour des raisons qui ne sont ni liées à l'orientationsexuelle ni à une déficience de la fonction utérine paraît tellement illégitime du point devue éthique qu'elle n'est même pas abordée. Dans ce contexte, la gestation pour autruiest le plus souvent envisagée comme une forme de procréation médicalement assistée etle point de vue médical occupe une place importante dans la discussion à ce sujet ${ }^{39}$.Lorsqu'il est invoqué, l'argument de la solidarité l'est d'ailleurs en lien avec ce diagnosticmédical d'infertilité, de même que le dispositif légal envisagé pour lui faire droit ${ }^{40}$.

Or, les arguments habituellement employés pour dénoncer l'immoralité de lagestation pour autrui ne suffisent pas à rendre compte de cette disqualification. Celle-ci a des sources qui sont ailleurs, dans un ensemble d'éléments compliqués à démêler d'avec les arguments courants sur la gestation pour autrui : un questionnement sur ce qu'est et doit être une « famille » ou une forme recevable de parentalité. La solidarité procréative dont lasociété française fait preuve à divers égards, et notamment dans l'organisation de sonsystème de soins, n'est donc pas sans limites ${ }^{41}$. Le problème n'est pas que des limitespuissent être posées, mais qu'elles sont à ce stade de la réflexion collectiveinsuffisamment explicitées et justifiées, même si une discussion a commencé à prendreforme ces dernières décennies, qui contribue à

\footnotetext{
${ }^{38}$ Selon une position élaborée dès le début des années 1980 (Un enfant à tout prix, Paris, Le Seuil, 1983), G. Delaisi de Parseval présente l'apport de la psychanalyse sur ce sujet. Elle y remet en cause l'idée selon laquelle grandir dans une famille créée par un couple de même sexe constituerait pour l'enfant un danger. Elle distingue la sexualité des parents - qui constitue un enjeu pour tout enfant, quelle que soit son orientation - d'autres questions liées au nombre de parents réunis autour de l'enfant et à la connaissance de sa filiation et de son mode de procréation. Selon elle, la gestation pour autrui peut être envisagée au bénéfice de couples homosexuels dès lors que l'accès à la filiation et au mode de procréation est garanti à l'enfant et que la mère porteuse soit aussi celle qui donne son ovocyte. V. Depadt-Sebag, pour sa part, est opposée à la gestation pour autrui pour les couples homosexuels dès lors que cette gestation est envisagée, ce qui est le cas dans ce rapport, comme une forme d'assistance médicale à la procréation. Cf. Accès à la parenté - Assistance médicale à la procréation et adoption, Rapport cité, p. 94 sqq.

${ }^{39}$ Cette orientation de la discussion éthique est par exemple lisible dans ce propos de l'Avis 110 pré-cité : «C'est pourquoi le présent avis s'interrogera à titre principal sur l'opportunité éthique d'ajouter la GPA à la liste des techniques d'AMP autorisées. Il est entendu que certains des arguments éthiques invoqués pourraient être étendus, mutatis mutandis, à la procréation pour autrui, celle qui peut être réalisée sans l'intervention du corps médical, à laquelle il sera parfois fait allusion sans cependant que lui soit consacrés des développements spécifiques ».

${ }^{40}$ Rapport Terra Nova: La réponse solidaire qu'il propose se veut «entourée d'un nombre maximum de précautions », soumettant à l'examen d'un comité d'experts les projets parentaux et les « candidatures » de mères porteuses. La décision de ce comité reposerait sur une appréciation au plan médical, psychologique et sociale et le respect d'un certain nombre de critères ; notamment, âge du couple, part génétique de l'acte procréatif (au moins un parent génétique de l'enfant), expérience de la maternité déjà faite pour la mère porteuse, information psychologique de la mère porteuse, accord de son conjoint, accord des différentes parties en présence sur l'absence d'anonymat, don si possible unique, dédommagement qui exclut toute forme de marchandisation et confère à l'acte une dimension de don, possibilité pour la mère porteuse d'interrompre la grossesse », p. 78 sqq. Voir aussi la proposition énoncée dans I. Théry et A.-M. Leroyer, Filiation, origines, parentalité - le droit face aux nouvelles valeurs de responsabilité générationnelle, Paris, O. Jacob, 2014, Annexe au chapitre 7, p. 211 sqq. ${ }^{41}$ Sur ce point, je me permets de renvoyer à mon ouvrage : M. Gaille, Le désir d'enfant - histoire intime, enjeu politique (Paris, PUF, 2011), chapitre 4.
} 
faire de la famille et de la parentalité desobjets de questionnement plutôt que des évidences intangibles ${ }^{42}$.

Comme le suggère l'analyse critique proposée dans la seconde partie, lesarguments qui circulent dans la discussion ne sont pas tous élaborés de façon rigoureuse. Ils ne sont pas tous convaincants, notamment celui de l'instrumentalisation.Et leur effet est parfois à double tranchant. Par exemple, le désaveu moral évoqué plushaut peut avoir deux traductions inverses dans la sphère politique et juridique :l'interdiction ou l'encadrement juridique de la gestation pour autrui. En effet, il peut toutautant justifier la première que le second dans un contexte procréatif globalisé, «post-westphalien» pour reprendre l'expression de la philosophe américaine N. Fraser ${ }^{43}$. À partir du moment où l'on estime que le droit a d'abord pour vocation de protéger lesplus faibles et les plus vulnérables, on peut interdire la gestation pour autrui, ou au contraire organiser sa pratique sur un plan légal dans l'espace national, afin d'éviter l'exploitation des mèresporteuses et éventuellement celles de personnes désireuses d'avoir un enfant, confrontées à un marché mondial « sauvage » ${ }^{44}$ de la procréation.

Enfin, il y a matière à approfondir certains éléments peu présents dans ladiscussion morale française, mais repérables ailleurs et dotés d'un poids important dans d'autres cultures morales, juridiques et politiques que celle de la France. En effet, nombre d'arguments sur la gestation pour autrui se rencontrent en effet dans plusieurs espaces culturels, linguistiques et nationaux, même si leurpoids respectifs diffère et les formulations varient d'un espace social, politique, juridiqueà l'autre ${ }^{45}$. Or, la réflexion française a moins investi qued'autres l'aspect financier de la gestation pour autrui. Cela tient certainement à l'état dudroit qui interdit la commercialisation du corps humain (comme totalité, du moins) etdans ses capacités reproductives. Cette disposition juridique semble faire consensus.Pour ceux qui s'aventurent à défendre la légalisation de la gestation pour autrui, il va desoi qu'elle doit relever de la logique du don, ou tout au plus être indemnisée. De ce fait,l'analyse des dispositifs juridiques qui légalisent une forme consentie, contractualisée,non fondée sur des motifs de profit économique de gestation pour autrui, est peufréquente et il faut l'«importer»d'autres contextes pour l'introduire dans la discussion et en examiner les différentes facettes morales. ${ }^{46}$ De ce fait également, l'interrogation du philosophe américain $\mathrm{M}$. Sandel à l'égard des effets sociaux, moraux etcivilisationnels de l'extension tous azimuts de la logique de

\footnotetext{
${ }^{42}$ A. Cadoret, Des parents comme les autres? Homosexualité et parenté, Paris, O. Jacob, 2002 ; M. Godelier, Les métamorphoses de la parenté, Paris, Flammarion, 2010 ; M. Gross, Parent ou Homo : faut-il choisir ? Idées reçues sur l'homoparentalité, éd. du Cavalier bleu, 2014. Les travaux issus des sciences historiques et sociales sur la famille mettent également en cause l'idée qu'il existe une conception univoque et universellement recevable de la famille. De son côté, A. M. Leroyer souligne l'absence d'une définition claire de la famille dans le droit français, dans Droit de la famille, PUF, 2011.

${ }^{43}$ N. Fraser, Scales of justice, Reimagining Political Space in a Globalizing World, Malden, Polity Press, 2008.

${ }^{44}$ B. Steinbock, «SurrogateMotherhood as Prenatal Adoption», art. cit., p. 49; D. Mehl, Enfants du don Procréation médicalement assistée - parents et enfants témoignent, op. cit., p. 307 ; J. Merchant, «Une gestation pour autrui «éthique» est possible », art. cit.

${ }^{45}$ Dès 1984, le Warnock Report britannique identifia des enjeux que l'on retrouve peu ou prou discutés dans l'ensemble de la littérature, en France et ailleurs, consacrée à la gestation pour autrui : M. Warnock, Report of the Committee of Inquiry into Human Fertilisation and Embryology, Londres, Her Majesty's Stationarey Office, 1984. Les quatre enjeux énoncés dans ce rapport sont : le respect de la dignité humaine au vu de la dimension financière de la gestation pour autrui ; la relation entre mère porteuse/enfant à naitre ; la commercialisation des êtres humains (l'accent est mis ici sur la vente de l'enfant plutôt que sur la location d'utérus) : les risques liés à toute grossesse.

${ }^{46}$ J. Merchant, «Une gestation pour autrui 'éthique' est possible », art. cit.
} 
marché a du mal à se faire entendre. ${ }^{47}$ Or cette interrogation pourrait nourrir la réflexion sur la gestation pour autrui, en particulier lorsqu'on l'envisage dans sa dimension transnationale.

\footnotetext{
${ }^{47}$ M. Sandel, Ce que l'argent ne saurait acheter, trad. fr.Christian Clerc, Paris, Le Seuil, 2014 (What money can't buy - The moral limits of markets, 2012). Je me permets sur ce point de renvoyer à l'article «Contre la marchandisation du corps: quels arguments pour quelle critique? », Raison publique, http://www.raisonpublique.fr/article712.html, parution le 12 décembre 2014. Les travaux de D. Dickenson (Body Shopping; converting body parts to profit, Oneworld Publications, 2009) et D. L. Spar, Baby Business : How Money, Science, and Politics Drive the Commerce of Conception (Cambridge, Mass. Harvard Business SchoolPress, 2006), donnent un aperçu concret de l'économie globale de la procréation, dont la gestation pour autrui n'est qu'un des volets.
} 